ARTICLE

https://doi.org/10.1038/s41467-020-19752-3

\title{
OPEN
}

\section{The epitaxy of 2D materials growth}

\author{
Jichen Dong ${ }^{1}$, Leining Zhang ${ }^{1,2}$, Xinyue Dai ${ }^{1,3}$ \& Feng Ding (1) ${ }^{1,2 凶}$
}

Two dimensional (2D) materials consist of one to a few atomic layers, where the intra-layer atoms are chemically bonded and the atomic layers are weakly bonded. The high bonding anisotropicity in 2D materials make their growth on a substrate substantially different from the conventional thin film growth. Here, we proposed a general theoretical framework for the epitaxial growth of a 2D material on an arbitrary substrate. Our extensive density functional theory (DFT) calculations show that the propagating edge of a 2D material tends to align along a high symmetry direction of the substrate and, as a conclusion, the interplay between the symmetries of the $2 \mathrm{D}$ material and the substrate plays a critical role in the epitaxial growth of the 2D material. Based on our results, we have outlined that orientational uniformity of $2 \mathrm{D}$ material islands on a substrate can be realized only if the symmetry group of the substrate is a subgroup of that of the 2D material. Our predictions are in perfect agreement with most experimental observations on 2D materials' growth on various substrates known up to now. We believe that this general guideline will lead to the large-scale synthesis of wafer-scale single crystals of various $2 \mathrm{D}$ materials in the near future.

\footnotetext{
${ }^{1}$ Centre for Multidimensional Carbon Materials, Institute for Basic Science, Ulsan 44919, Korea. ${ }^{2}$ School of Materials Science and Engineering, Ulsan National Institute of Science and Technology, Ulsan 44919, Korea. ${ }^{3}$ School of Materials Science and Engineering, Shandong University, 250061 Jinan, China.

$凶_{\text {email: f.ding@unist.ac.kr }}$
} 
T wo-dimensional (2D) materials are potentially the most promising materials for future device applications but in practice, wafer-scale single crystals of various $2 \mathrm{D}$ materials are needed to realize these applications ${ }^{1-4}$. Recently, the seamless coalescence of millions of well-aligned islands of a 2D material epitaxially grown on a substrate has been successfully used to synthesize wafer-scale single crystals of graphene $e^{5-7}$, hexagonal boron nitride ${ }^{8,9}$, and $\mathrm{MoS}_{2}{ }^{10}$. This strategy is expected to be generalized to grow various $2 \mathrm{D}$ single crystals in the near future. Nevertheless, the unique behavior of $2 \mathrm{D}$ materials growth, different from that predicted by classical theory of epitaxy, necessitates the development of a general theory for the epitaxial growth of $2 \mathrm{D}$ materials ${ }^{5-22}$.

In graphene CVD growth, the zigzag (ZZ) edge is generally the slowest propagating edge because of its highest barrier for edge propagation ${ }^{23,24}$ and the alignment of graphene on a substrate has been broadly observed to be dependent on the symmetry of the substrate. For example, graphene islands grown on a $\mathrm{Cu}(111)$ or $\mathrm{Cu}(110)$ surface are well-aligned but those grown on a $\mathrm{Cu}(100)$ surface are observed to be along two perpendicular directions ${ }^{5,11,12}$. Among the three low-index $\mathrm{Cu}$ surfaces, highly robust alignment of graphene can be obtained on the $\mathrm{Cu}(111)$ surface and currently, the principal method of graphene single crystal production is by epitaxially growing graphene single crystals on $\mathrm{Cu}(111)$ surface $^{5-7}$.

Because of the lower $\mathrm{C}_{3 \mathrm{~V}}$ symmetry of $\mathrm{hBN}$, the alignment of $\mathrm{hBN}$ on a substrate is different from that of graphene. Three of its six $\mathrm{ZZ}$ edges are nitrogen terminated and the other three are boron terminated (named as ZZN and ZZB edges hereafter). In most experiments, the ZZN edge has been proven to be the slowest propagating and kinetic Wulff construction leads to triangular hBN islands enclosed by three ZZN edges ${ }^{25-27}$. In contrast to epitaxial graphene growth, well-aligned $\mathrm{hBN}$ islands have rarely been observed. When grown on $\mathrm{Cu}(111)$ or $\mathrm{Cu}(110)$ surfaces, triangular hBN islands aligned along two opposite directions were found ${ }^{13-15,28}$, while those grown on $\mathrm{Cu}(100)$ surfaces had four different orientations ${ }^{14}$. Recent works have shown that well-aligned hBN islands can be successfully achieved by using a $\mathrm{Cu}$ substrate with tailored step edges, thus enabling epitaxial growth of wafer-scale hBN single crystals ${ }^{8,9}$.

Similar to hBN, most transition metal dichalcogenides (TMDCs) possess three-fold symmetry and present very similar epitaxial behavior on substrates; for example, with two possible alignments on $\mathrm{Au}(111)^{16}, \mathrm{Al}_{2} \mathrm{O}_{3}(0001)^{17-19}$, and $\mathrm{GaN}(0001)^{20}$ surfaces. Well-aligned $\mathrm{WS}_{2}$ islands have been grown on $\mathrm{hBN}$ surface $^{21}$ and nearly well-aligned $\mathrm{WSe}_{2}$ islands have been grown on a vicinal $\mathrm{Al}_{2} \mathrm{O}_{3}(0001)$ surface 22 . Most recently, centimeter scale single-crystalline $\mathrm{MoS}_{2}$ was obtained by the coalescence of well-aligned $\mathrm{MoS}_{2}$ grains on a vicinal $\mathrm{Au}(111)$ surface ${ }^{10}$.

All these experimental observations strongly indicate that the alignment of a $2 \mathrm{D}$ material on a substrate depends on both its symmetry and that of the substrate and a general theory for $2 \mathrm{D}$ materials epitaxy that helps to predict the alignment of various $2 \mathrm{D}$ materials on different substrates is highly desirable to serve as a guideline for experimental design.

Here, based on extensive density functional theory (DFT) calculations, we present a general theory to explain how the alignment of a $2 \mathrm{D}$ material on a substrate is intimately related to its interaction with the substrate and how the epitaxial growth of the 2D material on a substrate is critically dependent on the interplay of the symmetries of the 2D material and the substrate. Our theory explains most known experimental observations on 2D material epitaxial growth and hence can serve as a guideline for the experimental synthesis of various $2 \mathrm{D}$ single crystals, as well as $2 \mathrm{D}$ polycrystalline materials with designed grain boundaries.

\section{Results}

2D material-substrate interaction and the alignment of a 2D material on a substrate. There are hundreds of important $2 \mathrm{D}$ materials and the possible substrate types are also of the same order of magnitude. So, it is impossible to calculate the interactions of all possible combinations of $2 \mathrm{D}$ materials and substrates, which is greater than 100,000 . Without losing the generality, we can classify the interactions between $2 \mathrm{D}$ materials and various substrates into two sceneries:

(i) The edge of the $2 \mathrm{D}$ material is terminated by the substrate, such as graphene or hBN on an active metal substrate, where the strong interaction between the edge of the $2 \mathrm{D}$ material and the pristine substrate facet determines the alignment of the $2 \mathrm{D}$ material and its epitaxial growth behavior ${ }^{29,30}$;

(ii) The edge of the 2D material is self-passivated or terminated by active atoms from the environment of its growth, such as $\mathrm{H}$ or $\mathrm{OH}$ groups ${ }^{31-34}$, where the weak interaction between the bulk of the $2 \mathrm{D}$ material and the pristine substrate facet dominates the alignment of the 2D material.

To establish an epitaxial relationship between a $2 \mathrm{D}$ material and a substrate for scenery (i), we firstly explore the interaction between the edges of graphene or hBN with the three low-index $\mathrm{Cu}$ surfaces, namely $\mathrm{Cu}(111), \mathrm{Cu}(100)$, and $\mathrm{Cu}(110)$. The calculated binding energies between a graphene $(\mathrm{hBN}) \mathrm{ZZ}$ (ZZN) edge on the three low-index Cu surfaces as a function of the angle of edge alignment that is defined as the angle between the edge and a $\mathrm{Cu}\langle 110\rangle$ direction of the substrate, are shown in Fig. 1 (please refer to method section and Supplementary Figs. 1-6 in Section 1 of supplementary information (SI) for more details on the calculation and modeling). We clearly see that on each of the three low-index surfaces, the strongest binding energy appears when the $\mathrm{ZZ}(\mathrm{ZZN})$ edge of graphene $(\mathrm{hBN})$ is along a $\mathrm{Cu}\langle 110\rangle$ direction of the surface; the difference between the binding energy minimum and maximum is significant, $>0.2 \mathrm{eV}$ per edge atom. Hence, on a $\mathrm{Cu}$ surface, a well-aligned small graphene or hBN island of $\sim 2 \mathrm{~nm}$ (which has only $\sim 200$ atoms of which $\sim 40$ are at the edge) has an energy advantage of $>8 \mathrm{eV}$ over misaligned ones. This binding energy difference is large enough to maintain a growing graphene or hBN island in a well-aligned configuration on a Cu surface.

To elucidate the reason behind the strongest binding of a $\mathrm{ZZ}$ (ZZN) edge along the $\langle 110\rangle$ direction on a $\mathrm{Cu}$ surface, we have plotted the electron density distributions of $\mathrm{Cu}(111), \mathrm{Cu}(100)$, and $\mathrm{Cu}(110)$ surfaces, respectively, in Fig. $2 \mathrm{a}-\mathrm{c}$. It can be seen that on all the three surfaces, the isosurface fluctuation in electron density is the lowest along the $\langle 110\rangle$ direction, indicating that the close-packed $\langle 110\rangle$ atomic rows form a pattern with alternative ridges and valleys of uniform height on the surface. In a straight $\mathrm{ZZ}(\mathrm{ZZN})$ edge of graphene(hBN), the less stable edge atoms form a straight line and this straight edge is preferentially passivated by either a ridge or a valley of the $\mathrm{Cu}$ surface instead of crossing over ridges and valleys on the surface, which results in distortion of the edge. To further illustrate preferential passivating of the graphene $\mathrm{ZZ}$ edge by a close-packed atomic row, we compare the atomic structures of the interfaces and the charge density differences of the graphene edge along both $\langle 110\rangle$ and other directions on the three low-index Cu surfaces (Fig. $2 \mathrm{~d}-\mathrm{i}$ ). When the graphene $\mathrm{ZZ}$ edge is aligned along the $\mathrm{Cu}\langle 110\rangle$ direction, all the edge atoms are well passivated by a $\mathrm{Cu}\langle 110\rangle$ atomic row and the edge remains straight. In contrast, if the graphene edge is along another direction, some of the edge atoms are poorly passivated and the edge is no longer straight because of the fluctuating 
a

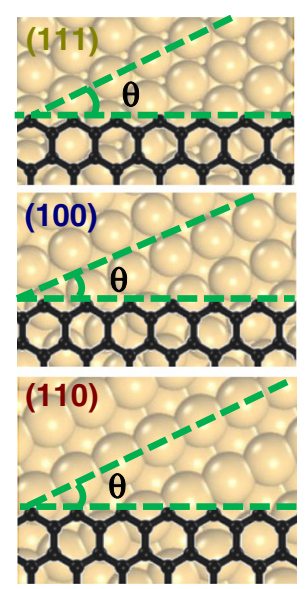

C

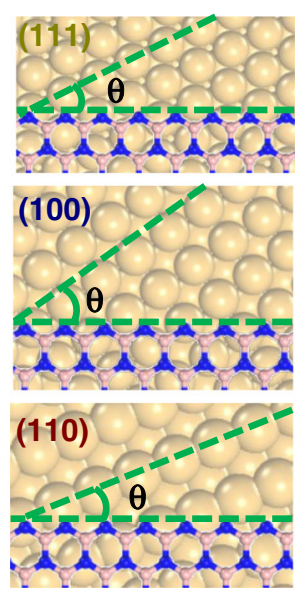

b

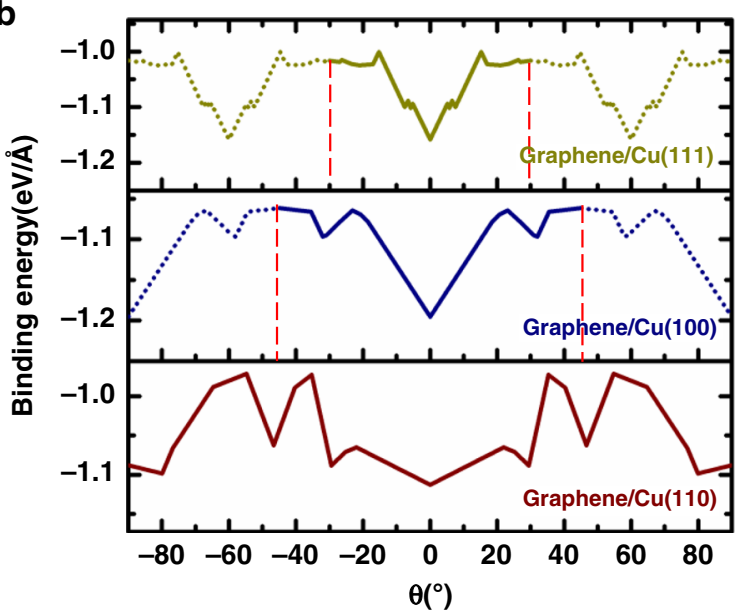

d

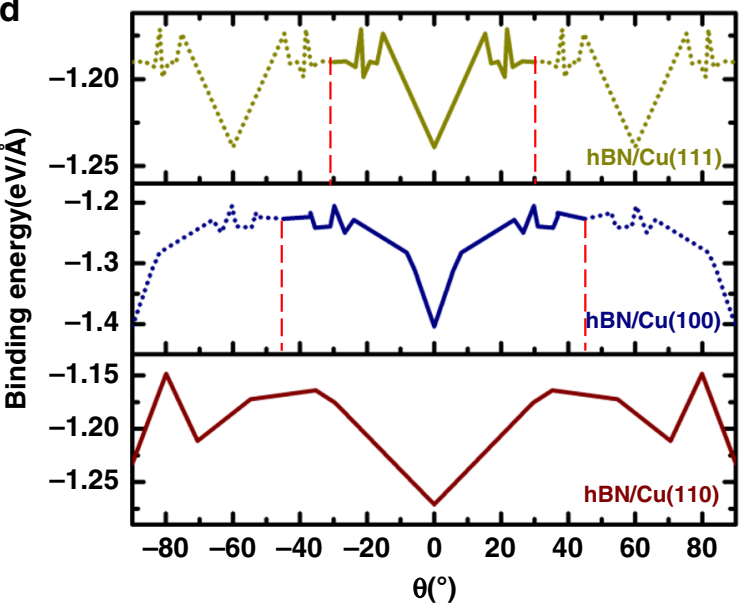

Fig. 1 Binding energy of $\mathbf{Z Z ( Z Z N )}$ edge of graphene(hBN) on three low-index Cu surfaces. Model of a graphene ZZ edge on three low-index Cu surfaces $\mathbf{a}$ and the calculated binding energies of the edge on the three substrates as a function of the alignment angle $\mathbf{b}$. Model of a ZZN edge of hBN on the three low-index Cu surfaces $\mathbf{c}$ and the calculated binding energies of the edge on the three substrates as a function of the alignment angle $\mathbf{d}$. Vertical lines highlight the periodicity of the binding energy curves.

ridge-valley pattern of the surface. The above analysis clearly shows the superiority of the close packed direction of a substrate in passivating a high-symmetric edge in a $2 \mathrm{D}$ material.

Our analysis of a straight graphene/hBN edge preferring the direction of valley or ridge of the isosurface of $\mathrm{Cu}$ substrate can be generally applied to most combinations of $2 \mathrm{D}$ materials on various substrates. It is noted that the lattice constant of graphene/hBN ZZ edge matches that of the $\langle 110\rangle$ direction of $\mathrm{Cu}$ substrate well. Thus, it is worth to consider a system without perfect lattice-match. To address the effect of lattice-mismatch, we consider the interaction between a $\mathrm{ZZ}$ edge of graphene and the $\mathrm{Pt}(111)$ surface, where the lattice constant of graphene $\mathrm{ZZ}$ edge is about $12.6 \%$ smaller than that of $\mathrm{Pt}\langle 110\rangle$ direction. The binding energies of a graphene $\mathrm{ZZ}$ edge on the $\mathrm{Pt}(111)$ surface as a function of the alignment angle of the graphene edge are shown in Supplementary Fig. 7. As expected and similar to that of a graphene $\mathrm{ZZ}$ edge on $\mathrm{Cu}(111)$ surface, the graphene $\mathrm{ZZ}$ edge prefers the alignment along a $\langle 110\rangle$ direction of the $\mathrm{Pt}$ (111) surface. As an example, above calculation suggests that the conclusion that the slowest propagating (also highsymmetric) edge of a $2 \mathrm{D}$ material prefers to align along the high-symmetric direction of an active metal substrate, regardless of the lattice-match between the $2 \mathrm{D}$ material and the substrate.
If the edge of a $2 \mathrm{D}$ material is self-terminated, such as the selenium-terminated edges of TMDC materials ${ }^{33}$, or terminated by the $\mathrm{H}$ or $\mathrm{OH}$ functional groups, such as the edges of graphene or hBN grown on a less active metal substrate $31,32,34$. In such a scenery, the interaction between the $2 \mathrm{D}$ material edge and the substrate is no longer very strong and the dominating interaction is the weak interaction between the bulk of the $2 \mathrm{D}$ material and the substrate. To elucidate the alignment of a $2 \mathrm{D}$ material on a substrate for scenery (ii), we calculate the interaction between $\mathrm{hBN}$ and $\mathrm{Cu}(111$ ) (Fig. 3a, b, and Supplementary Fig. 8) and $\mathrm{Au}(111)$ (Supplementary Fig. 9) surfaces, respectively, by using the periodic boundary condition models and the interaction between a triangular $\mathrm{WS}_{2}$ cluster and the hBN surface (Fig. 3c, d and Supplementary Fig. 10). All these calculations, together with previous studies of graphene on $\mathrm{Cu}(111)$ surface ${ }^{35}$ and $\mathrm{MoS}_{2}$ on $\mathrm{Al}_{2} \mathrm{O}_{3}(0001)$ surface ${ }^{36}$, show that a high-symmetric direction of a $2 \mathrm{D}$ material ( $\mathrm{ZZ}$ directions of graphene, hBN and TMDCs) prefers to align along the highsymmetric directions of a substrate, such as the $\langle 110\rangle$ directions of $\mathrm{Cu}(111)$ and $\mathrm{Au}(111)$ surfaces, and $\langle 11 \overline{2} 0\rangle$ direction of hBN and $\mathrm{Al}_{2} \mathrm{O}_{3}(0001)$ surfaces.

The above results allow us to draw a conclusion of the alignment of a $2 \mathrm{D}$ material on an arbitrary surface, i.e., a highsymmetric direction of the $2 \mathrm{D}$ island prefers to align along a high-symmetric direction of the substrate. Although we just 
a

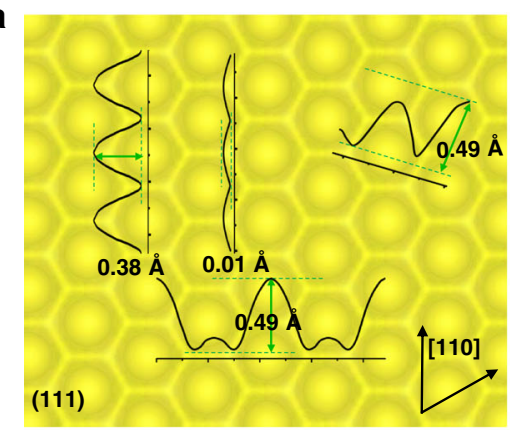

d

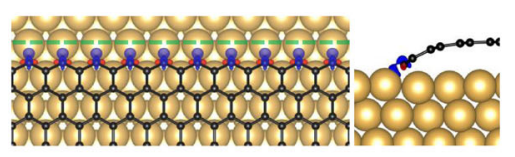

e

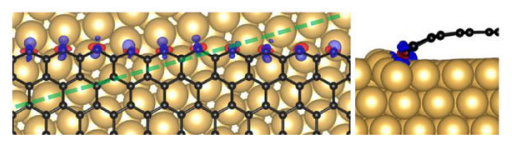

b
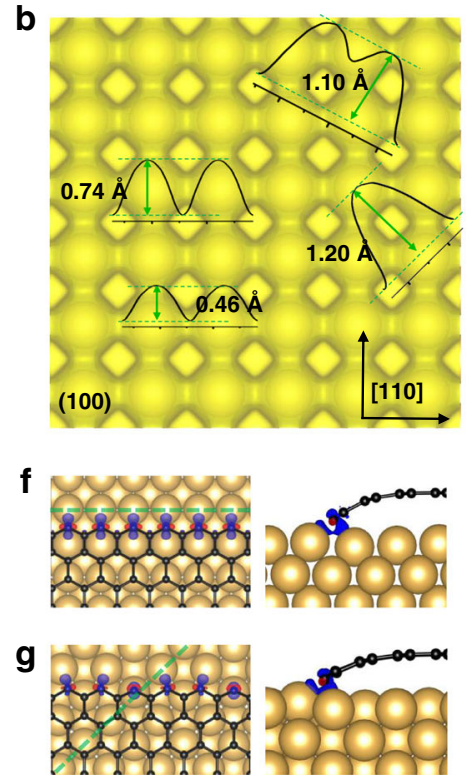

C

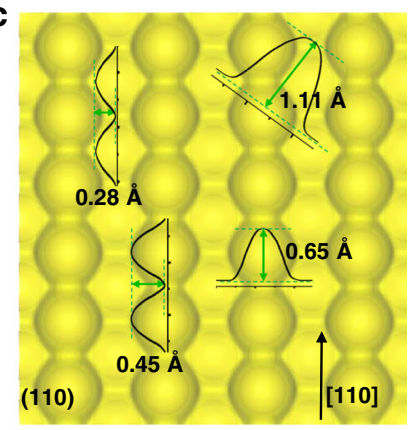

h
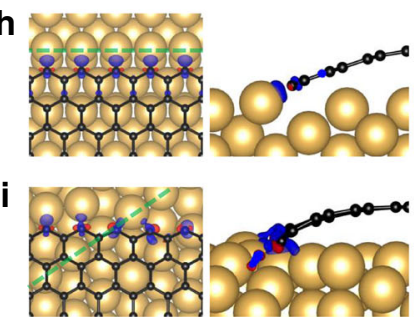

Fig. 2 Charge density analysis of low-index Cu surfaces and zigzag edges of graphene adsorbed on them with different orientations. a-c The electron density profiles with isovalue of $0.03 \mathrm{Bohr}^{-3}$ of $\mathrm{Cu}(111), \mathrm{Cu}(100)$, and $\mathrm{Cu}(110)$ surfaces, respectively. The height of the isosurface along different directions of the different $\mathrm{Cu}$ surfaces are shown. The $\mathrm{Cu}[110]$ directions on these surfaces are also marked. $\mathbf{d}$-i Charge density differences with isovalue of $0.01 \mathrm{Bohr}^{-3}$ of graphene on $\mathrm{Cu}(111) / \mathrm{Cu}(100 / \mathrm{Cu}(110))$ surfaces with misorientation angles of $0^{\circ} \mathbf{d}, \mathbf{f}, \mathbf{h}$ and $15.3^{\circ} / 45^{\circ} / 35.3^{\circ} \mathbf{e}, \mathbf{g}$, i. Blue and red colors in $\mathbf{d}$-i represent electron accumulation and depletion, respectively.

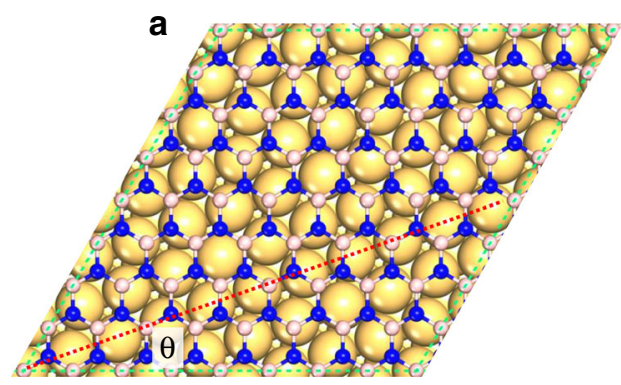

C

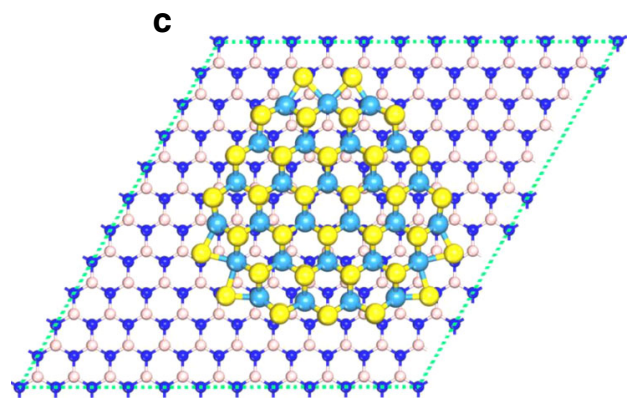

b

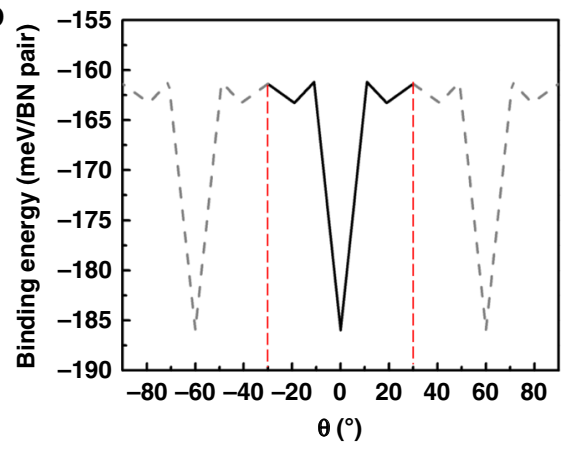

d

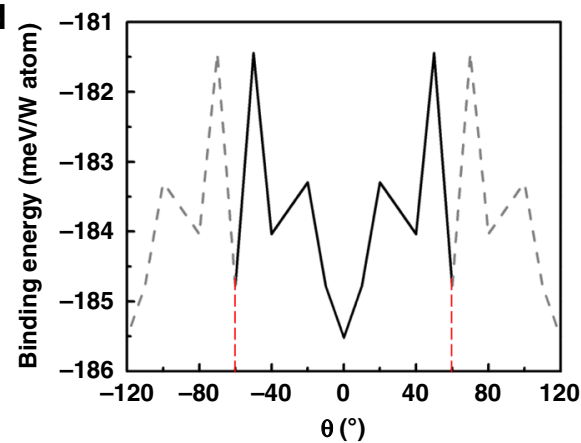

Fig. 3 The calculated interactions between the bulks of 2D materials and substrates. Periodic boundary condition model of hBN on the $\mathrm{Cu}(111)$ surface a and the calculated binding energies of $\mathrm{hBN}$ on the $\mathrm{Cu}(111)$ surface as a function of the alignment angle $(\theta)$ between the $\mathrm{ZZ}$ direction of hBN lattice and a $\langle 110\rangle$ direction of the $\mathrm{Cu}(111)$ surface $\mathbf{b}$. Cluster model of a WS $\mathrm{W}_{2}$ cluster on the hBN surface $\mathbf{c}$ and the calculated binding energies of the WS $\mathrm{S}_{2}$ cluster on the $\mathrm{hBN}$ surface as a function of the alignment angle $(\theta)$ between the $\mathrm{ZZ}$ direction of $\mathrm{WS}_{2}$ and the $\mathrm{ZZ}$ direction of the $\mathrm{hBN}$ surface $\mathbf{d}$.

explored a very limited systems of 2D materials grown on various substrates, as will be seen later, this rule is in accordance with most experimental observations on the epitaxial growth of various $2 \mathrm{D}$ materials, such as the CVD synthesis of graphene, $\mathrm{hBN}$, and TMDCs on various transition metals or nonmetallic substrates (please refer to Section 2 in SI). Thus, we believe that this rule can be applied for the epitaxial growth of various $2 \mathrm{D}$ materials on different substrates.

The alignment of $2 \mathrm{D}$ materials on various substrates. Having established the principle that determines the alignment of a $2 \mathrm{D}$ 


\section{Table 1 The number of equivalent but different orientations of a 2D material on a substrate based on the interplay between their} symmetries.

\begin{tabular}{|c|c|c|c|c|c|c|c|c|c|c|c|c|c|c|c|c|}
\hline $\begin{array}{l}\mathbf{G}_{\mathbf{2 D}} \\
\mathbf{G}_{\text {Sub }}\end{array}$ & \multicolumn{4}{|l|}{$\mathrm{C}_{6 \mathrm{~V}}$} & \multicolumn{4}{|l|}{$C_{4 v}$} & \multicolumn{4}{|l|}{$C_{3 v}$} & \multicolumn{4}{|l|}{$C_{2 v}$} \\
\hline $\begin{array}{l}\mathbf{G}_{\text {Sub }} \\
\mathbf{G}_{\text {2D@Sub }}\end{array}$ & $\begin{array}{l}C_{6 v} \\
C_{6 v}\end{array}$ & $\begin{array}{l}C_{4 v} \\
C_{2 v}\end{array}$ & $\begin{array}{l}C_{3 v} \\
C_{3 v}\end{array}$ & $\begin{array}{l}C_{2 v} \\
C_{2 v}\end{array}$ & $\begin{array}{l}C_{6 v} \\
C_{2 v}\end{array}$ & $\begin{array}{l}C_{4 V} \\
C_{4 V}\end{array}$ & $\begin{array}{l}C_{3 v} \\
C_{v}\end{array}$ & $\begin{array}{l}C_{2 v} \\
C_{2 V}\end{array}$ & $\begin{array}{l}C_{6 v} \\
C_{3 v}\end{array}$ & $\begin{array}{l}C_{4 v} \\
C_{v}\end{array}$ & $\begin{array}{l}C_{3 v} \\
C_{3 v}\end{array}$ & $\begin{array}{l}C_{2 v} \\
C_{v}\end{array}$ & $\begin{array}{l}C_{6 V} \\
C_{2 V}\end{array}$ & $\begin{array}{l}C_{4 V} \\
C_{2 V}\end{array}$ & $\begin{array}{l}C_{3 v} \\
C_{v}\end{array}$ & $\begin{array}{l}C_{2 v} \\
C_{2 v}\end{array}$ \\
\hline$\left|G_{\text {sub }}\right|$ & 12 & 8 & 6 & 4 & 12 & 8 & 6 & 4 & 12 & 8 & 6 & 4 & 12 & 8 & 6 & 4 \\
\hline$\left|G_{2 D @ s u b}\right|$ & 12 & 4 & 6 & 4 & 4 & 8 & 2 & 4 & 6 & 2 & 6 & 2 & 4 & 4 & 2 & 4 \\
\hline$N_{1}$ & 1 & 2 & 1 & 1 & 3 & 1 & 3 & 1 & 2 & 4 & 1 & 2 & 3 & 2 & 3 & 1 \\
\hline
\end{tabular}

Here, $G_{\text {sub }}, G_{2 D}$ and $G_{2 D @ S u b}$ are symmetry groups of the substrate, the $2 D$ material and the $2 D$ material-substrate system, $\left|G_{\text {sub }}\right|$ and $\left|G_{2 D @ s u b}\right|$ are the orders of $G_{\text {sub }}$ and $G_{2 D @ S u b}$; and $N_{1}=\mid G_{\text {sub }} / /$ $\left|G_{2 D @ s u b}\right|$ is the number of equivalent but different directions of the $2 \mathrm{D}$ material on the substrate.

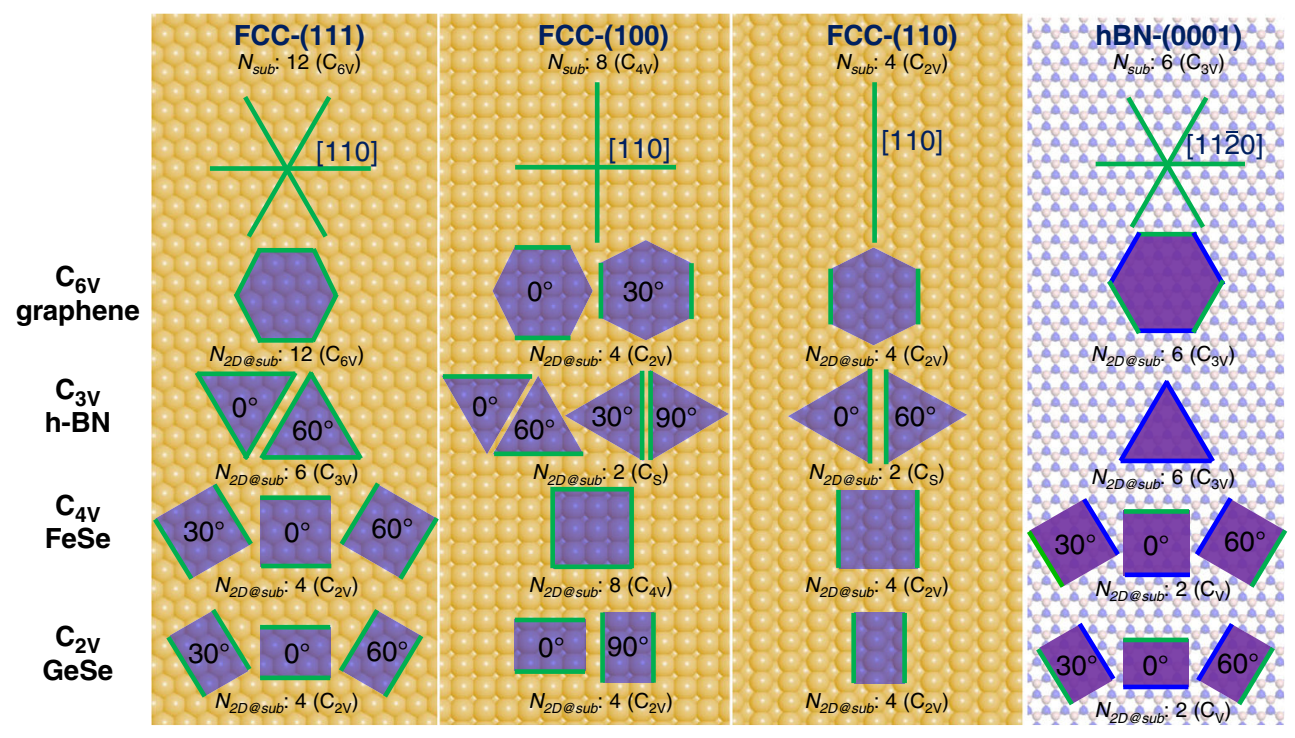

Fig. 4 Alignment of single-crystalline 2D islands with various symmetries on low-index fcc surfaces and hBN (0001) surface. The $\langle 110\rangle$ or $\langle 11 \overline{2} 0\rangle$ crystallographic orientations of substrates are denoted by green lines. 2D islands are represented by purple polygons. The symmetry groups of the $2 \mathrm{D}$ material, substrate, and the system of the 2D island on the substrate are provided. The number of different symmetry operations of the substrate and the whole system are $N_{\text {sub }}$ and $N_{2 D} @$ sub, respectively. The nitrogen and boron atoms are represented by blue and pink spheres, respectively. The relative anticlockwise misorientation angles of grains with different orientations are given. Due to the $\mathrm{C}_{3 \mathrm{~V}}$ symmetry of hBN, the edges of a $2 \mathrm{D}$ material, which are parallel to hBN $\langle 11 \overline{2} 0\rangle$ directions, have different local environments and not equivalent, and thus are denoted by blue and green lines, respectively.

material on a substrate, we proceed to discuss the interplay between the symmetry of a $2 \mathrm{D}$ material and that of the substrate in epitaxial growth. Let us consider a $2 \mathrm{D}$ material with a $\mathrm{G}_{2 \mathrm{D}}$ symmetry group on a substrate with a symmetry group of $G_{\text {sub }}$. The symmetry group of the whole system, $\mathrm{G}_{2 \mathrm{D} @ \text { Sub }}$, must be a subgroup of either $\mathrm{G}_{2 \mathrm{D}}$ or $\mathrm{G}_{\text {Sub }}$ because any symmetry operation of $\mathrm{G}_{2 \mathrm{D} @ \text { Sub }}$ will not change the alignment of the $2 \mathrm{D}$ material or the substrate. As shown in SI, we have proved that the number of equivalent but different directions of a $2 \mathrm{D}$ material on a substrate can be calculated by

$$
N_{1}=\frac{\left|\mathrm{G}_{\mathrm{sub}}\right|}{\left|\mathrm{G}_{2 \mathrm{D} @ \text { sub }}\right|},
$$

where $\left|G_{\text {sub }}\right|$ and $\left|G_{2 D @ s u b}\right|$ are the orders, or numbers of different symmetry operations, of $\mathrm{G}_{\text {sub }}$ and $\mathrm{G}_{2 \mathrm{D} @ \text { sub }}$, respectively.

According to the principle of $2 \mathrm{D}$ materials alignment discussed in the previous paragraph, the presence of a highsymmetry edge of a $2 \mathrm{D}$ material along a high-symmetry direction of the substrate ensures that the symmetry group of the whole system, $G_{2 D @ S u b}$, is the largest subgroup of both $G_{\text {sub }}$ and $\mathrm{G}_{2 \mathrm{D}}$. We have considered various combinations of the symmetries of the $2 \mathrm{D}$ material and the substrate and the numbers of equivalent but different alignments of various $2 \mathrm{D}$ materials are shown in Table 1.

Without loss of generality, we have used fcc(111), fcc(100), fcc (110), and $\mathrm{hBN}(0001)$ surfaces as different examples of a substrate with 6-, 4-, 2- and 3-fold symmetries to illustrate the alignment of various $2 \mathrm{D}$ materials on them. Figure 4 presents the various ways in which $2 \mathrm{D}$ materials with 2-, 3-, 4- and 6-fold symmetries are aligned on these substrates. From the figure, we can deduce that in order to keep the whole system with the highest symmetry, there are:

$1,2,1$ and 1 equivalent but different alignments for a 6-fold symmetric 2D material on 6-, 4-, 2- and 3-fold symmetric substrates;

$2,4,2$, and 1 equivalent but different alignments for a 3 -fold symmetric 2D material on 6-, 4-, 2- and 3-fold symmetric substrates;

$3,1,1$ and 3 equivalent but different alignments for a 4 -fold symmetric 2D material on 6-, 4-, 2- and 3-fold symmetric substrates;

3, 2, 1 and 3 equivalent but different alignments for a 2 -fold 


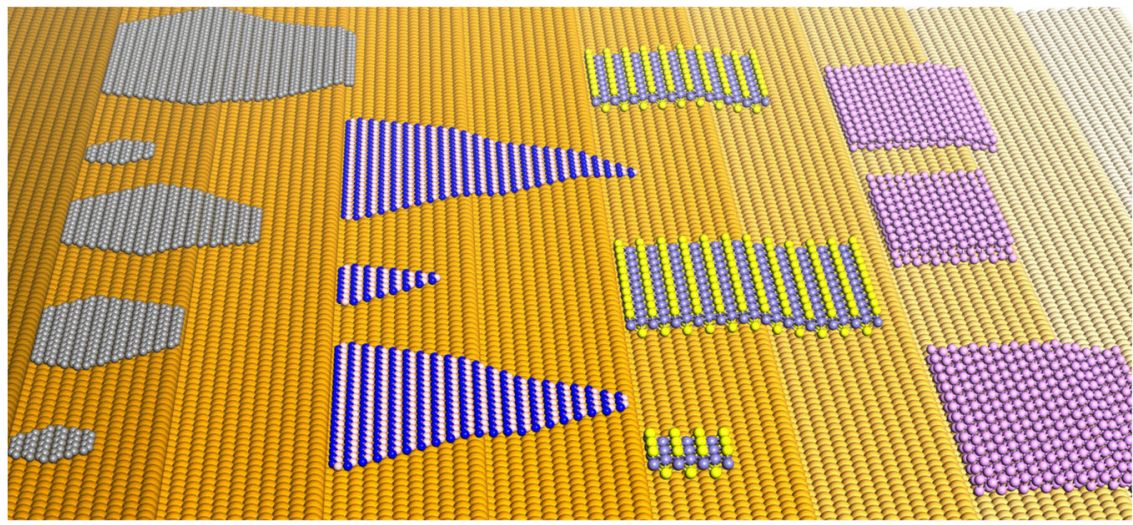

Fig. 5 Schematics showing well-aligned 2D materials (from left to right are graphene, hBN, FeSe and black phosphorene, respectively.) on a vicinal fcc (111) surface. One edge of each island is passivated by a high-symmetric step edge of the substrate.

symmetric 2D material on 6-, 4-, 2- and 3-fold symmetric substrates, respectively.

The number of equivalent but different alignments of various $2 \mathrm{D}$ materials on substrates of different symmetries are shown in Fig. 4 and these numbers are in perfect agreement with the symmetry analysis shown in Table 1 . Besides the number of equivalent but different alignments of a $2 \mathrm{D}$ material on a substrate, Fig. 4 also gives the misalignment angles of equivalent islands of $2 \mathrm{D}$ materials. On the substrates with 6-, 4-, 3-, 2-fold symmetries, the misalignment angles are $\frac{i}{3} \pi, \frac{i}{2} \pi, \frac{2 i}{3} \pi, i \pi, i=1,2,3, \ldots$ respectively.

It is important to note that a high-symmetric edge of a $2 \mathrm{D}$ material along a high-symmetric direction of a substrate is critical for the above analysis. If the high-symmetric edge of the 2D material is along a low-symmetric direction of the substrate with mirror symmetry, the symmetry group $\mathrm{G}_{2 \mathrm{D} @ \text { Sub }}$ will have no mirror symmetry and the least number of equivalent but different alignments of the $2 \mathrm{D}$ material will be 2, which makes orientational uniformity impossible.

Comparison with experimental observations. We have summarized most of known experimental obversions on 2D materials epitaxial growth, including graphene growth on low-index $\mathrm{Cu}$ surfaces (Supplementary Table 1), hBN growth on low-index $\mathrm{Cu}$ surfaces (Supplementary Table 2), TMDCs growth on various low-index substrates, including $\mathrm{Al}_{2} \mathrm{O}_{3}, \mathrm{Au}, \mathrm{GaN}, \mathrm{hBN}$ (Supplementary Table 3 ), and various $2 \mathrm{D}$ material grown on different high-index substrates (Supplementary Table 4). It is interesting to note that there is a perfect agreement between the experimentally observed numbers of alignments and misalignment angles of 2D materials on these substrates with those predicted by our theoretical analysis. Hence, we believe that the epitaxial relationship is valid for the epitaxial growth of most $2 \mathrm{D}$ materials grown on different substrates.

Strategies toward the epitaxial growth of various 2D single crystals. From Eq. (1), we can see that once the symmetry group of the substrate, $G_{S u b}$, is a subgroup of the $2 D$ material, $G_{2 D}$, or in other words

$$
\mathrm{G}_{\text {Sub }} \subset \mathrm{G}_{2 \mathrm{D}}
$$

the symmetry group of the system $\mathrm{G}_{2 \mathrm{D} @ \text { Sub }}$ could be same as

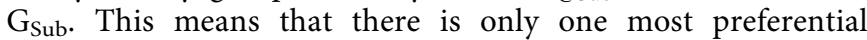
alignment of the $2 \mathrm{D}$ material on the substrate and the orientational uniformity of a large number of islands of the 2D material is possible. As shown in Table 1 and Fig. 4, this can be applied to 2D materials with 6-fold symmetry, such as graphene on a $6-, 3$ - or 2 -fold symmetric substrate, $2 \mathrm{D}$ materials with 4-fold symmetric on a 4- or 2-fold symmetric substrate, 3 -fold symmetricy $2 \mathrm{D}$ materials on a substrate with 3 -fold symmetry, and a 2 -fold symmetric $2 \mathrm{D}$ material on a 2 -fold symmetric substrate. Up to now, the seamless stitching of well-aligned graphene islands have been realized on $\mathrm{Cu}(111)$ and $\mathrm{Ge}(110)$ surfaces, both of which are in accordance with the above described analysis ${ }^{5-7,37,38}$.

Among the thousands of known 2D materials, most of them have the 3 -fold symmetry, such as the most explored hBN and TMDCs. As shown above, a substrate with 3-fold symmetry is expected to be suitable for the epitaxial growth of $2 \mathrm{D}$ materials with a three-fold symmetry, but it is difficult to find proper lowindex substrates with 3 -fold symmetry. Although some low-index substrates has the 3 -fold symmetry, such as the $\mathrm{Cu}(111)$ surface and the $\mathrm{Al}_{2} \mathrm{O}_{3}(0001)$ surface, the atoms of the top layer of the substrate generally have a higher symmetry, such as the atoms of the top $\mathrm{Cu}(111)$ layer have the 6-fold symmetry. So, for a $\mathrm{C}_{3 \mathrm{~V}} 2 \mathrm{D}$ material on a $\mathrm{C}_{3 \mathrm{~V}}$ substrate, there are generally two deep local minima in the formation profile which corresponds two high symmetric configurations, such as the $0^{\circ}$ and $60^{\circ}$ configurations of a $\mathrm{WS}_{2}$ on the hBN surface shown in Fig. $3 \mathrm{~d}$ and Supplementary Fig. 10, and the $0^{\circ}$ and $60^{\circ}$ configurations of $\mathrm{MoS}_{2}$ on the $\mathrm{Al}_{2} \mathrm{O}_{3}$ (0001) surface in Fig. $3 \mathrm{~h}$ of ref. ${ }^{34}$ (please refer to Supplementary Fig. 11 for the configuration difference). Experimentally, antiparallel TMDC islands on 3-fold symmetric substrates are generally seen and parallel aligned TMDC islands could be realized by precise control of the experimental condition (Supplementary Table 3) ${ }^{17-21}$. From Eq. (2), we can see that if the substrate has the $\mathrm{C}_{1}$ symmetry group, the condition for epitaxial growth can be satisfied for any $2 \mathrm{D}$ materials, implying that on a substrate with no symmetry, we may be able to achieve orientational uniformity for any 2D materials. In practice, substrates with very low symmetry could be a high-index surface or a vicinal surface of a low-index surface. As illustrated in Fig. 5, a high-index surface has a large number of low-index terraces connected by parallel step edges. These step edges of the substrate can serve as nucleation sites to initiate the growth of the $2 \mathrm{D}$ material. Furthermore, these step edges interact preferentially with an edge of the $2 \mathrm{D}$ material to promote the orientational uniformity of the $2 \mathrm{D}$ material. In this manner, the orientational uniformity of various 2D materials has been widely observed. As listed in Supplementary Table 4, epitaxial growth of well-aligned $\mathrm{hBN}$ islands have been observed on $\mathrm{Cu}(102), \mathrm{Cu}(103)$, and vicinal $\mathrm{Cu}(110)$ surfaces ${ }^{39,40}$, where one of the three edges of the triangular $\mathrm{hBN}$ island is aligned along the step edge of the substrate. In addition, well-aligned $\mathrm{WSe}_{2}$ islands were also observed on $\mathrm{Al}_{2} \mathrm{O}_{3}(0001)$ surface with step edges ${ }^{22}$. Recently, such a strategy has been used to grow wafer scale single- 
crystalline $\mathrm{hBN}$ on vicinal $\mathrm{Cu}(110)$ surface and $\mathrm{Cu}(111)$ surface with step egdes ${ }^{8,9}$, and centimeter scale single-crystalline $\mathrm{MoS}_{2}$ on an $\mathrm{Au}(111)$ surface with aligned step edges ${ }^{10}$. DFT calculations in these studies have shown that the weak interaction between the bulk of the $2 \mathrm{D}$ material and the substrate and/or the strong interaction between the edge of 2D material and step edge of the substrate lead to the favorable alignment of the $2 \mathrm{D}$ islands along the step edges of the substrate ${ }^{8-10,39,40}$. Since high-index surfaces can be easily obtained by miscutting a single crystal, we believe that this could be a general strategy for the synthesis of various $2 \mathrm{D}$ materials in the future.

\section{Discussion}

We would like to note that the fact that a high symmetric direction of a $2 \mathrm{D}$ material prefers to align along a high symmetric direction of a substrate, which is revealed by our extensive DFT calculations in this study, is the foundation of our main conclusion. Otherwise, orientational uniformity of a $2 \mathrm{D}$ material on a substrate, even the symmetry group of the substrate is a subgroup of that of the 2D material, cannot be realized. Currently, most previous studies on the synthesis of large-sized single crystalline hBN and $\mathrm{MoS}_{2}$ employed vicinal (111) or (110) surfaces with parallel step edges ${ }^{8-10}$, where $2 \mathrm{D}$ materials tend to align along these step edges and the general study on the epitaxial 2D materials growth on various high index surface is very rare. Besides the vicinal surfaces which are close to one of the lowindex surfaces, our study also predicts that the high-index surfaces that are largely deviated from all the low-index surfaces, such as the (123) surface of an fcc material, are also ideal for the epitaxial growth of large-scale single-crystalline $2 \mathrm{D}$ materials. Furthermore, our theory provides a principle to determine the alignment of a 2D material on any given substrates and, thus, it offers a strategy of synthesizing 2D materials with well-defined grain boundaries, for instance, polycrystalline graphene with grain boundaries of a $30^{\circ}$ misorientation angle can be synthesized on an $f c c(100)$ surface, similar to the case of graphene growth on a liquid $\mathrm{Cu}$ surface ${ }^{41}$.

In conclusion, our study clearly demonstrates that the interplay of the symmetries of the $2 \mathrm{D}$ material and the substrate is critical for the epitaxial growth of $2 \mathrm{D}$ materials. Both theoretical analysis and experimental observations show that a high-symmetric direction of a 2D material tends to be aligned along a high-symmetric direction of a substrate, so that the $2 \mathrm{D}$ material-substrate system has the highest possible symmetry. Based on the symmetry analysis and the rules for the preferential alignment of a 2D material on a substrate, we established a library of the different possible alignments of various $2 \mathrm{D}$ materials on different substrates to serve as a guideline for experimental design. Furthermore, we theoretically proved that the epitaxial growth of a $2 \mathrm{D}$ single crystal can be realized only if the symmetry group of the substrate is a subgroup of that of the $2 \mathrm{D}$ material. To meet the requirement for single-crystalline $2 \mathrm{D}$ material growth, we propose using substrates with high-index surfaces which have lower symmetry to template the epitaxial growth of various $2 \mathrm{D}$ materials; this strategy has been successfully demonstrated (Nature 570, 91 (2019); Nature 579, 219 (2020); ACS nano 14, $5036(2020))$ and is in agreement with experimental observations. After submission of this manuscript, we have noticed that same strategy has been employed for the epitaxial synthesis of singlecrystalline nanoribbons of TMDCs (Nat. Mater. DOI 10.1038/ s41563-020-0795-4 (2020)), which further validated the proposed approach of synthesizing large area single-crystal 2D materials. Our study thus provides a theoretical foundation for the synthesis of wafer-scale $2 \mathrm{D}$ single crystals.

\section{Methods}

DFT calculations of edge binding energies. All DFT calculations were carried out via the Vienna ab initio simulation Package (VASP) ${ }^{42,43}$. The exchange-correlation effect was treated by the Perdew-Burke-Ernzerhof generalized gradient approximation (GGA) ${ }^{44}$. The interaction between valence electrons and ion cores was described by the projected augmented wave (PAW) method ${ }^{45}$ and the k-point mesh was sampled by a separation of $0.03 \AA^{-1}$

To calculate the binding energy of a graphene $\mathrm{ZZ}$ edge to $\mathrm{Cu}(111),(100)$, and (110) surfaces, $\mathrm{Cu}$ slabs consisting of three (111), (100), or (110) atomic layers were constructed to mimic the $\mathrm{Cu}$ substrates. Graphene nanoribbons along the $\mathrm{ZZ}$ direction, which were three hexagons wide and one of the two $\mathrm{ZZ}$ edges passivated by hydrogen, were constructed. Because of the incommensurate lattice constants between the graphene $\mathrm{ZZ}$ nanoribbon and the $\mathrm{Cu}$ substrates, only a small number of periodic structures can be constructed with a graphene $\mathrm{ZZ}$ nanoribbon adsorbed on low-index $\mathrm{Cu}$ surfaces along different directions, of which the number of atoms is not too large and can be handled by DFT calculations, as shown in Supplementary Figs. 1-3. The initial distance between the graphene $\mathrm{ZZ}$ nanoribbon and the $\mathrm{Cu}$ substrate is set to $3.06 \AA$, which was estimated from DFT-D2 calculations ${ }^{46}$, and it is the typical equilibrium distance between a graphene layer and the underlying $\mathrm{Cu}$ substrate surface. Structure optimization was conducted with the atomic positions of the lowest $\mathrm{Cu}$ atomic layer fixed. In addition, the vertical positions of carbon atoms that are passivated by hydrogen were also fixed during structure optimization. To eliminate the imaginary interaction between periodic images along the vertical direction, a vacuum layer with a $15 \AA$ thickness was used to separate the Cu slabs. All the structures were relaxed until the force on each unfixed atom was $<0.01 \mathrm{eV} / \AA$, with an energy convergence of $10^{-4} \mathrm{eV}$.

In a similar manner, $\mathrm{hBN}$ nanoribbons along the $\mathrm{ZZ}$ direction were also constructed with their ZZB edges passivated by hydrogen. The structures with hBN ZZ ribbons adsorbed on low-index Cu substrate surfaces are shown in Supplementary Figs. 4 and 6. The distance between hBN nanoribbon and the Cu substrate is set to be $3.10 \AA$, which was obtained by optimizing a hBN sheet on $\mathrm{Cu}(111)$ surface by DFT-D2 calculations ${ }^{46}$. During structure optimization, the vertical positions of boron atoms passivated by hydrogen were fixed. In addition, the atomic positions of the lowest $\mathrm{Cu}$ atomic layer were also fixed.

The method similar to above calculations was also employed to calculate the binding energies between the graphene $\mathrm{ZZ}$ edge and $\mathrm{Pt}(111)$ surface. The calculated structures are shown in Supplementary Fig. 7. as

The binding energy of a ZZ edge of graphene or hBN to the substrate is defined

$$
E_{\mathrm{b}}=\frac{E_{\text {total }}-E_{\text {sub }}-E_{\text {ribbon }}}{l},
$$

where $E_{\text {ribbon }}, E_{\text {sub }}$, and $E_{\text {total }}$ are, respectively, the energies of the nanoribbon, the substrate and the total energy of the nanoribbon adsorbed on the substrate; $L$ is the edge length of the nanoribbon.

To calculate the weak interaction between $\mathrm{hBN}$ wall and $\mathrm{Cu}(111)$ or $\mathrm{Au}(111)$ surface, a hBN layer was stacked to a $\mathrm{Cu}(111)$ or $\mathrm{Au}(111)$ slab consisting of three atomic layers under periodic boundary condition and with different alignment angles. The calculated structures are provided in Supplementary Figs. 8 and 9. During structure optimization, the bottom atomic layer of the metal slab was fixed The binding energy between the hBN wall and the metal substrate is defined as

$$
E_{\mathrm{b}}=\frac{E_{\text {total }}-E_{\mathrm{hBN}}-E_{\mathrm{sub}}}{N_{\mathrm{BN}}},
$$

where $E_{\mathrm{total}}, E_{\mathrm{hBN}}$, and $E_{\text {sub }}$ are the energies of the whole system, the hBN layer and the substrate, respectively. $N_{\mathrm{BN}}$ is the number of $\mathrm{BN}$ pairs of the hBN layer in the unit cell of the whole system.

To calculate the weak interaction between $\mathrm{WS}_{2}$ and $\mathrm{hBN}$ layer, a triangular $\mathrm{WS}_{2}$ cluster consisting of $60 \mathrm{~S}$ atoms and $27 \mathrm{~W}$ atoms was placed on a hBN layer with different orientations (Supplementary Fig. 10). Because the edges of the $\mathrm{WS}_{2}$ cluster are passivated by $\mathrm{S}$ and the hBN layer is chemically inert, the interaction between the $\mathrm{WS}_{2}$ cluster and the hBN layer should be dominated by the $\mathrm{WS}_{2}$ wall and the $\mathrm{hBN}$ layer. The binding energy between the $\mathrm{WS}_{2}$ cluster and the $\mathrm{hBN}$ layer is defined as

$$
E_{\mathrm{b}}=\frac{E_{\text {total }}-E_{\mathrm{hBN}}-E_{\mathrm{WS}_{2}}}{N_{\mathrm{W}}},
$$

where $E_{\text {total }}, E_{\mathrm{hBN}}$, and $E_{\mathrm{WS} 2}$ are the energies of the whole system, the hBN layer and the $\mathrm{WS}_{2}$ cluster, respectively. $N_{\mathrm{W}}$ is the number of $\mathrm{W}$ atoms in the $\mathrm{WS}_{2}$ cluster.

\section{Data availability}

The data that support the findings of this study are available from the corresponding authors on reasonable request.

Received: 5 July 2020; Accepted: 19 October 2020; Published online: 17 November 2020 


\section{References}

1. Geim, A. K. \& Novoselov, K. S. The rise of graphene. Nat. Mater. 6, 183-191 (2007).

2. Watanabe, K., Taniguchi, T. \& Kanda, H. Direct-bandgap properties and evidence for ultraviolet lasing of hexagonal boron nitride single crystal. Nat. Mater. 3, 404-409 (2004).

3. Zhou, J. et al. A library of atomically thin metal chalcogenides. Nature 556 355-359 (2018).

4. Song, C.-L. et al. Molecular-beam epitaxy and robust superconductivity of stoichiometric FeSe crystalline films on bilayer graphene. Phys. Rev. B 84, 020503 (2011).

5. $\mathrm{Xu}, \mathrm{X}$. et al. Ultrafast epitaxial growth of metre-sized single-crystal graphene on industrial $\mathrm{Cu}$ foil. Sci. Bull. 62, 1074-1080 (2017).

6. Deng, B. et al. Wrinkle-free single-crystal graphene wafer grown on strainengineered substrates. ACS Nano 11, 12337-12345 (2017).

7. Nguyen, V. L. et al. Seamless stitching of graphene domains on polished copper (111) foil. Adv. Mater. 27, 1376-1382 (2015).

8. Wang, L. et al. Epitaxial growth of a 100-square-centimetre single-crystal hexagonal boron nitride monolayer on copper. Nature 570, 91-95 (2019)

9. Chen, T.-A. et al. Wafer-scale single-crystal hexagonal boron nitride monolayers on $\mathrm{Cu}$ (111). Nature 579, 219-223 (2020).

10. Yang, P. et al. Epitaxial growth of centimeter-scale single-crystal MoS2 monolayer on Au (111). ACS Nano 14, 5036-5045 (2020).

11. Murdock, A. T. et al. Controlling the orientation, edge geometry, and thickness of chemical vapor deposition graphene. ACS Nano 7, 1351-1359 (2013).

12. Ogawa, Y. et al. Domain structure and boundary in single-layer graphene grown on $\mathrm{Cu}(111)$ and $\mathrm{Cu}(100)$ films. J. Phys. Chem. Lett. 3, 219-226 (2012).

13. Wood, G. E. et al. van der Waals epitaxy of monolayer hexagonal boron nitride on copper foil: growth, crystallography and electronic band structure. 2D Mater. 2, 025003 (2015).

14. Song, X. et al. Chemical vapor deposition growth of large-scale hexagonal boron nitride with controllable orientation. Nano Res. 8, 3164-3176 (2015).

15. Uchida, Y., Iwaizako, T., Mizuno, S., Tsuji, M. \& Ago, H. Epitaxial chemical vapour deposition growth of monolayer hexagonal boron nitride on a $\mathrm{Cu}$ (111)/sapphire substrate. Phys. Chem. Chem. Phys. 19, 8230-8235 (2017).

16. Grønborg, S. S. et al. Synthesis of epitaxial single-layer MoS2 on Au(111). Langmuir 31, 9700-9706 (2015).

17. Zhang, $X$. et al. Diffusion-controlled epitaxy of large area coalesced WSe2 monolayers on sapphire. Nano Lett. 18, 1049-1056 (2018).

18. Aljarb, A. et al. Substrate lattice-guided seed formation controls the orientation of 2D transition-metal dichalcogenides. ACS Nano 11, 9215-9222 (2017).

19. Dumcenco, D. et al. Large-area epitaxial monolayer MoS2. ACS Nano 9, 4611-4620 (2015).

20. Ruzmetov, D. et al. Vertical 2D/3D semiconductor heterostructures based on epitaxial molybdenum disulfide and gallium nitride. ACS Nano 10, 3580-3588 (2016).

21. Lee, J. S. et al. Wafer-scale single-crystal hexagonal boron nitride film via selfcollimated grain formation. Science 362, 817-821 (2018).

22. Chen, L. et al. Step-edge-guided nucleation and growth of aligned WSe2 on sapphire via a layer-over-layer growth mode. ACS Nano 9, 8368-8375 (2015).

23. Dong, J., Zhang, L. \& Ding, F. Kinetics of graphene and $2 \mathrm{D}$ materials growth. Adv. Mater. 31, 1801583 (2019).

24. Ma, T. et al. Edge-controlled growth and kinetics of single-crystal graphene domains by chemical vapor deposition. Proc. Natl Acad. Sci. USA 110, 20386-20391 (2013).

25. Sekerka, R. F. Equilibrium and growth shapes of crystals: how do they differ and why should we care? Cryst. Res. Technol. 40, 291-306 (2005).

26. Zhang, Z., Liu, Y., Yang, Y. \& Yakobson, B. I. Growth mechanism and morphology of hexagonal boron nitride. Nano Lett. 16, 1398-1403 (2016).

27. Stehle, Y. Y. et al. Anisotropic etching of hexagonal boron nitride and graphene: question of edge terminations. Nano Lett. 17, 7306-7314 (2017).

28. Tay, R. Y. et al. Synthesis of aligned symmetrical multifaceted monolayer hexagonal boron nitride single crystals on resolidified copper. Nanoscale $\mathbf{8}$, 2434-2444 (2016)

29. Yuan, Q., Yakobson, B. I. \& Ding, F. Edge-catalyst wetting and orientation control of graphene growth by chemical vapor deposition growth. J. Phys. Chem. Lett. 5, 3093-3099 (2014).

30. Zhang, X., Xu, Z., Hui, L., Xin, J. \& Ding, F. How the orientation of graphene is determined during chemical vapor deposition growth. J. Phys. Chem. Lett. 3, 2822-2827 (2012).

31. Dong, J. et al. Formation mechanism of overlapping grain boundaries in graphene chemical vapor deposition growth. Chem. Sci. 8, 2209-2214 (2017).

32. Ren, X. et al. Grain boundaries in chemical-vapor-deposited atomically thin hexagonal boron nitride. Phys. Rev. Mater. 3, 014004 (2019).
33. Sang, X. et al. In situ edge engineering in two-dimensional transition metal dichalcogenides. Nat. Commun. 9, 2051 (2018).

34. Shu, H., Chen, X. \& Ding, F. The edge termination controlled kinetics in graphene chemical vapor deposition growth. Chem. Sci. 5, 4639-4645 (2014)

35. Dong, J., Zhang, L., Zhang, K. \& Ding, F. How graphene crosses a grain boundary on the catalyst surface during chemical vapour deposition growth. Nanoscale 10, 6878-6883 (2018).

36. Ji, Q. et al. Unravelling orientation distribution and merging behavior of monolayer MoS2 domains on sapphire. Nano Lett. 15, 198-205 (2015).

37. Jin, S. et al. Colossal grain growth yields single-crystal metal foils by contactfree annealing. Science 362, 1021-1025 (2018).

38. Lee, J.-H. et al. Wafer-scale growth of single-crystal monolayer graphene on reusable hydrogen-terminated germanium. Science 344, 286-289 (2014).

39. Li, J. et al. Growth of polar hexagonal boron nitride monolayer on nonpolar copper with unique orientation. Small 12, 3645-3650 (2016).

40. Wang, S. et al. Catalyst-selective growth of single-orientation hexagonal boron nitride toward high-performance atomically thin electric barriers. Adv. Mater. 31, 1900880 (2019).

41. Dong, J., Geng, D., Liu, F. \& Ding, F. Formation of twinned graphene polycrystals. Angew. Chem. Int. Ed. 58, 7723-7727 (2019).

42. Kresse, G. \& Hafner, J. Ab initio molecular dynamics for open-shell transition metals. Phys. Rev. B 48, 13115-13118 (1993).

43. Kresse, G. \& Furthmüller, J. Efficiency of ab-initio total energy calculations for metals and semiconductors using a plane-wave basis set. Comput. Mater. Sci. 6, 15-50 (1996).

44. Perdew, J. P., Burke, K. \& Ernzerhof, M. Generalized gradient approximation made simple. Phys. Rev. Lett. 77, 3865-3868 (1996).

45. Kresse, G. \& Joubert, D. From ultrasoft pseudopotentials to the projector augmented-wave method. Phys. Rev. B 59, 1758-1775 (1999).

46. Grimme, S. Semiempirical GGA-type density functional constructed with a long-range dispersion correction. J. Comput. Chem. 27, 1787-1799 (2006).

\section{Acknowledgements}

Authors acknowledge support from the Institute for Basic Science (IBS-R019-D1), South Korea. Computational resources from Cimulator, CMCM, IBS are also acknowledged.

\section{Author contributions}

F. Ding led the development of the concept and supervised the research. J. Dong, L. Zhang and X. Dai performed calculations and data analysis. J. Dong and F. Ding wrote the manuscript.

\section{Competing interests}

The authors declare no competing interests.

\section{Additional information}

Supplementary information is available for this paper at https://doi.org/10.1038/s41467020-19752-3.

Correspondence and requests for materials should be addressed to F.D

Peer review information Nature Communications thanks Yunqi Liu and the other, anonymous reviewer(s) for their contribution to the peer review of this work.

Reprints and permission information is available at http://www.nature.com/reprints

Publisher's note Springer Nature remains neutral with regard to jurisdictional claims in published maps and institutional affiliations.

Open Access This article is licensed under a Creative Commons Attribution 4.0 International License, which permits use, sharing, adaptation, distribution and reproduction in any medium or format, as long as you give appropriate credit to the original author(s) and the source, provide a link to the Creative Commons license, and indicate if changes were made. The images or other third party material in this article are included in the article's Creative Commons license, unless indicated otherwise in a credit line to the material. If material is not included in the article's Creative Commons license and your intended use is not permitted by statutory regulation or exceeds the permitted use, you will need to obtain permission directly from the copyright holder. To view a copy of this license, visit http://creativecommons.org/ licenses/by/4.0/

(C) The Author(s) 2020 